\title{
Electric Field and Self-Heating Effects on the Emission Time of Iron traps in GaN HEMTs
}

\author{
Marcello Cioni, Student Member, IEEE, Nicolò Zagni, Student Member, IEEE, Luca Selmi, \\ Fellow, IEEE, Gaudenzio Meneghesso, Fellow, IEEE, Matteo Meneghini, Senior Member, \\ IEEE, Enrico Zanoni, Fellow, IEEE, and Alessandro Chini
}

\begin{abstract}
In this paper we separately investigate the role of electric field and device self-heating (SHE) in enhancing the charge emission process from Fe-related buffer traps $\left(0.52 \mathrm{eV}\right.$ from $\left.E_{c}\right)$ in AIGaN/GaN High Electron Mobility Transistors (HEMTs). The experimental analysis was performed by means of Drain Current Transient (DCT) measurements for either i) different dissipated power

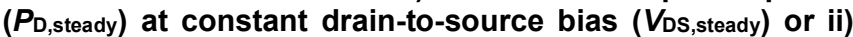

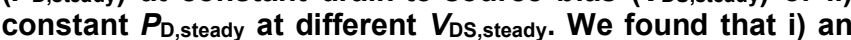

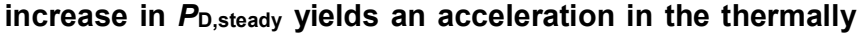
activated emission process, consistently with the temperature rise induced by SHE. On the other hand, ii) the field effect turned out to be negligible within the investigated voltage range, indicating the absence of Poole-Frenkel effect (PFE). A qualitative analysis based on the electric field values obtained by numerical simulations is then presented to support the interpretation and conclusions.
\end{abstract}

Index Terms- Gallium nitride, high electron mobility transistors (HEMTs), Poole-Frenkel effect (PFE), self-heating

\section{INTRODUCTION}

$\mathrm{A}$ $1 \mathrm{GaN} / \mathrm{GaN}$ high electron mobility transistors (HEMTs) are expected to play an important role in next generation RF communications systems, due to their high power and high frequency capability [1] that make them particularly interesting for the upcoming $5 \mathrm{G}$ revolution [2]. However, exploiting the whole potential of GaN-based power HEMTs still represents an open challenge due to dispersion phenomena limiting their performance and reliability [3]. In this regard, deep-levels related to intentional or undesired impurities incorporated during material growth or device fabrication induce trapping effects that severely affect the large signal operation. Iron $(\mathrm{Fe})$ dopants, for instance, are generally adopted in RF transistors [4,5] to obtain semi-

Manuscript received December 16, 2020. This work was partially supported by the Italian Ministry for University and Research (MIUR) under the PRIN 2017 Project "Empowering GaN-on-SiC and GaN-on$\mathrm{Si}$ technologies for the next challenging millimeter-wave applications".

M. Cioni, N. Zagni, L. Selmi and A. Chini are with Engineering Department "Enzo Ferrari", University of Modena and Reggio Emilia, Via P. Vivarelli, 10, 41125, Modena, Italy (e-mail: marcello.cioni@unimore.it).

G. Meneghesso, M. Meneghini and E. Zanoni are with Department of Information Engineering, University of Padova, via Gradenigo 6/B, Padova, 35131, Italy.

(c) 2021 IEEE. Personal use of this material is permitted. Permission from IEEE must be obtained for all other uses, in any current or future media, including reprinting/republishing this material for advertising or promotional purposes, creating new collective works, for resale or redistribution to servers or lists, or reuse of any copyrighted component of this work in other works. insulating buffer layers to increase the blocking voltage [6]. At the same time however, the deep-acceptor related to $\mathrm{Fe}$ dopants at $0.5-0.6 \mathrm{eV}$ from the conduction band minimum $\left(E_{\mathrm{C}}\right)$ [7-12] represents a major cause for Current Collapse (CC) in these devices [13], leading to a decreased output power and consequent reduction of Power Added Efficiency (PAE) [14]. These detrimental effects have led many authors to study the nature of Fe-trap in GaN (see [15] and references therein), but a precise definition of the physics behind this deep level is still an open issue [12]. Further studies are thus required to accurately model the Fe-trap response and, consequently, the device behavior. To this end, the study of the charge emission dynamics from traps represents an effective mean to gain insights on their physics. More precisely, the emission time constants of deep levels are strongly affected by the operating conditions in terms of bias, temperature and electric field distribution and these dependences are indicative of the traps nature. Aim of this paper is thus to further extend the knowledge on iron-related traps by investigating their emission rate dependence from temperature and electric field.

Concerning the role of temperature, it is well known that the emission of an electron from an impurity level to the conduction band is a thermally activated process with activation energy $E_{a}=E_{c}-E_{t}$ and emission time constant $\tau$, mutually related by the Arrhenius equation [16]. Accordingly, the net rate of carrier emission from traps is strongly temperature dependent, and this correlation is typically employed to extract $E_{a}$ from measurements at different ambient temperatures. However, the estimation of $E_{a}$ could be seriously affected by device self-heating (SHE) [17] which is known to raise the DUT temperature thus accelerating the emission process [17].

Electric field plays a similar role in enhancing the emission rate yielding a substantial reduction of the apparent activation energy [18]. Particularly, Poole-Frenkel Effect (PFE) is expected to dominate the field-enhanced emission for lowmedium voltage levels (i.e., conventional RF operations), while secondary effects like Phonon Assisted Tunneling (PAT) and Direct Tunneling (DT) come into play only for high field values (i.e., high voltages) [19]. However, PFE affects only charged Coulombic traps [20-22], making it difficult to predict its contribution without an in-depth 
knowledge of the trap state involved in the emission process.

To assess the dependence of the Fe-traps emission rate on SHE and electric field, we perform Drain Current Transient (DCT) measurements [23,24] starting from properly chosen bias conditions to evaluate these two concurrent effects separately. DCTs are thus monitored for either: i) different dissipated power $\left(P_{\mathrm{D} \text {,steady }}\right)$ with constant drain-to-source bias $\left(V_{\mathrm{DS} \text {,steady }}\right)$, or ii) different $V_{\mathrm{DS} \text {,steady }}$ with constant $P_{\mathrm{D} \text {,steady. The }}$ first condition allows investigating SHE while keeping constant the electric field; the second one, instead, allows determining the role of PFE without increasing SHE in the device. To the best of the authors knowledge, this is the first time in which PFE is studied for Fe-related buffer traps.

From the above analysis we find that increasing $P_{\mathrm{D} \text {,steady }}$ accelerates the thermally activated emission process from $\mathrm{Fe}$ traps, consistently with the temperature rise induced by SHE. Conversely, we find negligible dependence of the observed DCTs on varying electric field, suggesting the absence of PFE.

The rest of the paper is organized as follows. Section II provides a description of the Devices Under Test (DUTs) and their typical $I_{\mathrm{G}}-V_{\mathrm{GS}}, \quad I_{\mathrm{D}}-V_{\mathrm{GS}}, \quad I_{\mathrm{D}}-V_{\mathrm{DS}}$ and Power Sweep characteristics. Afterwards, a description of the measurement setup for DCTs used for trap characterization will follow. After proving the presence of dispersion effects connected to Fe-related traps within the tested devices, the emission rate dependence from SHE and electric field is investigated in Section III. In Section IV we report 2-D numerical simulations to estimate the electric field experienced by Fe-traps, while Section $\mathrm{V}$ reports a qualitative analysis on PFE, showing that, if present, PFE should have affected the emission rate within the investigated range. Finally, Section VI draws the conclusions of this work.

\section{DEVICE DESCRIPTION AND PRELIMINARY CHARACTERIZATION}

Devices tested in this work were single $\mathrm{AlGaN} / \mathrm{GaN}$ heterojunction HEMTs grown on SiC substrate (see Fig. 1). AlGaN barrier layer was $20 \mathrm{~nm}$ thick with a $22 \%$ Aluminum concentration while the $2 \mu \mathrm{m}$ thick GaN buffer was iron doped to obtain a semi-insulating layer [6]. Iron concentration in the $\mathrm{GaN}$ buffer starting from the $\mathrm{SiC}$ substrate interface is constant at $1 \times 10^{18} \mathrm{~cm}^{-3}$ until at a distance of $0.6 \mu \mathrm{m}$ from the $\mathrm{AlGaN} / \mathrm{GaN}$ interface where the iron concentration starts to decay with a slope of one decade every $0.4 \mu \mathrm{m}$. Device fabrication started with $\mathrm{Ti} / \mathrm{Al} / \mathrm{Ni} / \mathrm{Au}$ ohmic contact formation followed by device isolation by means of ion implantation. A $\mathrm{Si}_{3} \mathrm{~N}_{4}$ passivation layer was then deposited followed by a $\mathrm{CF}_{4}-$ based dry etching for the opening of the device gate foot. $\mathrm{Ni} / \mathrm{Pt} / \mathrm{Au}$ gate metallization was then evaporated self-aligned, yielding an I-shape gate contact without field-plates. Devices gate length was $0.5 \mu \mathrm{m}$ while gate-source and gate-drain spacings were $1 \mu \mathrm{m}$ and $2.5 \mu \mathrm{m}$, respectively. Three different gate peripheries were characterized, labelled as type A, B and $\mathrm{C}$, presenting a total gate width of $10 \times 100 \mu \mathrm{m}, 8 \times 75 \mu \mathrm{m}$ and $4 \times 75 \mu \mathrm{m}$ respectively.

Devices threshold voltage at $1 \mathrm{~mA} / \mathrm{mm}$ drain current level at $V_{\mathrm{DS}}=7 \mathrm{~V}$ was approximately $-2.8 \mathrm{~V}$ as shown in Fig. 2. For $V_{\mathrm{DS}}<60 \mathrm{~V}$, drain leakage current at $V_{\mathrm{GS}}=-6 \mathrm{~V}$ was below $\sim 20$

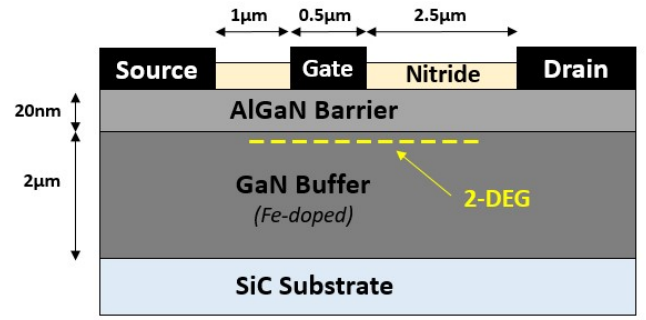

Fig. 1. Schematic structure of tested AIGaN/GaN HEMTs.
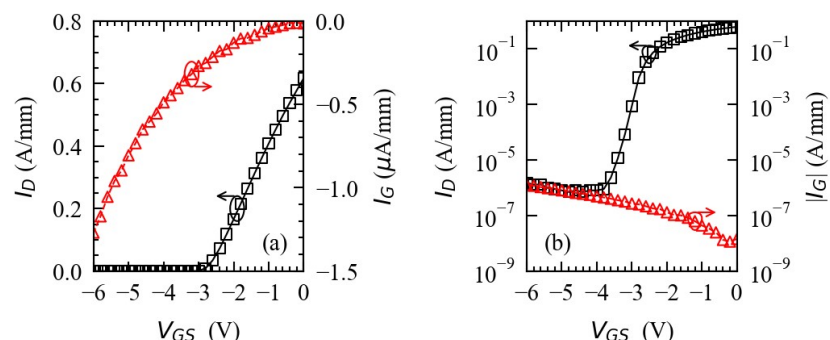

Fig. 2. (a) $I_{D}-V_{G S}\left(\right.$ at $V_{D S}=7 \mathrm{~V}$ ) and $I_{G}-V_{G S}$ characteristics for tested devices in linear scale. (b) $I_{D}-V_{G S}$ (at $V_{D S}=7 \quad \mathrm{~V}$ ) and $\left|I_{G}\right|-V_{G S}$ characteristics for tested devices in semi-log scale.
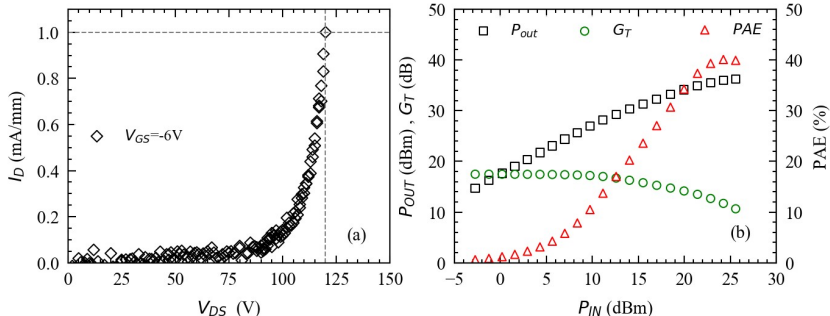

Fig. 3. (a) Breakdown characteristic for tested devices at $V_{G S}=-6$ V. (b) Power sweep characteristics measured at a frequency of $2.5 \mathrm{GHz}$ and quiescent drain bias voltage of $30 \mathrm{~V}$.

$\mu \mathrm{A} / \mathrm{mm}$ (not shown), whereas the breakdown voltage evaluated at $I_{\mathrm{D}}=1 \mathrm{~mA} / \mathrm{mm}$ [25] was about $120 \mathrm{~V}$, see Fig. 3(a).

In Fig. 3(b) we reported the results of $\mathrm{CW}$ load pull measurements performed on tested devices at $2.5 \mathrm{GHz}$ frequency and quiescent drain voltage of $30 \mathrm{~V}$. Output power $\left(P_{\text {OUT }}\right)$ reached a maximum of $4.1 \mathrm{~W} / \mathrm{mm}$ with a $40.1 \%$ peak power added efficiency (PAE).

Fig. 4 shows the typical $1 \mu \mathrm{s} / 100 \mu \mathrm{s}$ pulsed IV characteristics obtained on a type A device with two different quiescent baselines i.e., $\left(V_{\mathrm{GSq}}=0 \mathrm{~V}, V_{\mathrm{DSq}}=0 \mathrm{~V}\right)$ and $\left(V_{\mathrm{GSq}}=-3.8 \mathrm{~V}\right.$, $\left.V_{\mathrm{DSq}}=40 \mathrm{~V}\right)$. Saturated drain current $\left(I_{\mathrm{DSS}}\right)$ at $V_{\mathrm{DS}}=5 \mathrm{~V}$ is approximately $0.66 \mathrm{~A} / \mathrm{mm}$ when evaluated from the $(0 \mathrm{~V}, 0 \mathrm{~V})$ baseline while it decreases to $0.43 \mathrm{~A} / \mathrm{mm}$ when evaluated from the $(-3.8 \mathrm{~V}, 40 \mathrm{~V})$ bias, stemming from the presence of trapping phenomena leading to the degraded drain current swing.

The presence of Fe-doped buffer suggests that the observed current dispersion might be related to the response of ironrelated traps located within the buffer. The kinetics of said traps was thus investigated by means of drain current transient (DCT) measurements carried out at different temperatures.

DCTs induced by trapping phenomena have been measured with the double-pulse method sketched in Fig. 5, which allows to minimize the drain-source voltage $\left(V_{\mathrm{DS}}\right)$ variation during the transient [26]. The DUT is initially biased for few minutes in on-state conditions $\left(V_{\mathrm{DS} \text {,steady }}, V_{\mathrm{GS} \text {,steady }}\right)$ allowing it to reach a stable $I_{\mathrm{D} \text {,steady }}$ drain current level. A $1 \mu$ s trap-filling pulse is 


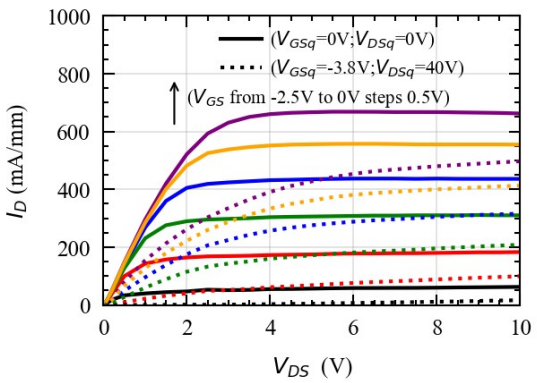

Fig. 4. Pulsed output characteristics from two different baselines: $V_{G S q}=0 \mathrm{~V}, V_{D S q}=0 \vee\left(\right.$ solid) and $V_{G S a}=-3.8 V, V_{D S q}=40 \vee$ (dotted). The current dispersion observed might be related to the response of ironrelated trap located within the buffer.

Drain Voltage VDS

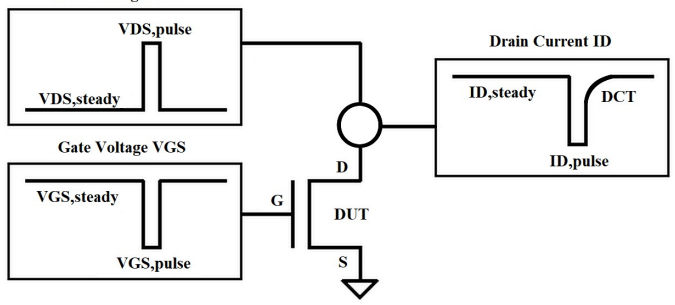

Fig. 5. Simplified schematic of the double-pulse method used for the DCTs acquisition. A custom pulser circuit fixes the potential at the drain terminal (D), whereas a waveform generator is used for pulsing $V_{G S}$. $V_{D S}$ and $I_{D}$ are measured with a digital sampling oscilloscope and the recovering of the drain current (DCT) is monitored over several time decades after the application of $1 \mu \mathrm{s}$ trap-filling pulse.

then applied with $V_{\mathrm{GS}, \text { pulse }}=-3.8 \mathrm{~V}$ and $V_{\mathrm{DS} \text {,pulse }}=8 \mathrm{~V}$, ensuring the occupation of the traps [13]. Then, the device is set back to the initial $\left(V_{\mathrm{DS} \text {,steady }}, V_{\mathrm{GS} \text {,steady }}\right)$ bias and the DCT is monitored over several time decades to allow for a full recovery of the initial $I_{\mathrm{D} \text {,steady }}$ drain current value.

DCTs were thus measured at $V_{\mathrm{DS} \text {,steady }}=4 \mathrm{~V}$ while $V_{\mathrm{GS} \text {,steady }}$ was settled in order to obtain a $50 \mathrm{~mA} / \mathrm{mm} I_{\text {D,steady }}$ current level. The apparently low values for both $V_{\mathrm{DS} \text {,steady }}$ and $I_{\mathrm{D} \text {,steady }}$ have been selected in order to reduce the influence of device self-heating which is known to affect the estimation of $E_{a}$ [17].

Typical DCTs obtained on a type A device in the $30{ }^{\circ} \mathrm{C}$ to $70{ }^{\circ} \mathrm{C}$ temperature range are depicted in Fig. 6(a). In order to evaluate the charge emission dynamics, DCTs have been fitted by a stretched multiexponential function [27]. The corresponding $\mathrm{d} I_{\mathrm{D}} / \mathrm{dlog}_{10} t$ signals are reported in figure $6(\mathrm{~b})$ where a single and well-defined peak can be observed. The associated emission time constant $(\tau)$ has been then extracted in correspondence to the peak obtained in the $\mathrm{d} I_{\mathrm{D}} / \mathrm{dlog}_{10} t$ signal, yielding the Arrhenius plot reported in the inset of Fig. 6(b). The linear fit of the points on the Arrhenius plot yielded a $0.52 \mathrm{eV}$ activation energy and $5 \times 10^{-16} \mathrm{~cm}^{2}$ cross section, which are consistent with values reported in the literature for Fe-related buffer traps [7-12]. To better identify the trap type, we compared the Arrhenius plot obtained in this work, with those obtained in previous reports and associated with Fe-traps [8,11,12,28-30], see Fig. 7. As we can see from Fig. 7, the activation energy, trap cross section and emission times are consistent with those largely reported for iron traps in $\mathrm{GaN}$. According to this observation and to the presence of a highly Fe-doped buffer layer, we can speculate that the monitored DCTs are due to the emission of electrons from traps related to iron dopants [8]. The devices tested in this paper can thus be used as test vehicles for the characterization of the iron trap
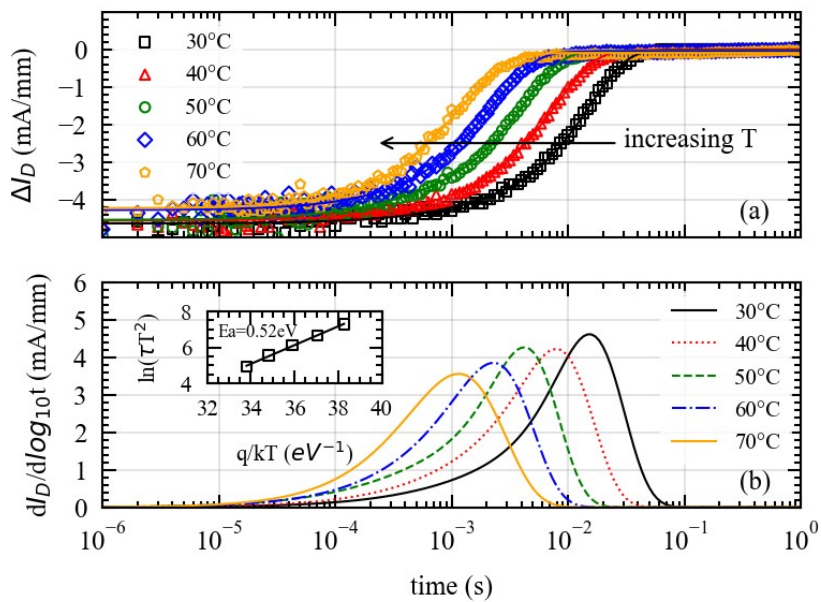

Fig. 6. (a) DCTs measured at different ambient temperatures (from 30 ${ }^{\circ} \mathrm{C}$ to $70^{\circ} \mathrm{C}$ ) for device $\mathrm{A}$. We represent the current variation $\left(\Delta I_{D}\right)$ with respect to the steady state current value at the end of the transient (/D,steady). (b) $d /_{D} / d_{l o g} \log _{10}$ used for drawing the Arrhenius plot (figure inset). The linear fit of the logarithmic form of the Arrhenius equation yields an activation energy $E a=0.52 \mathrm{eV}$.

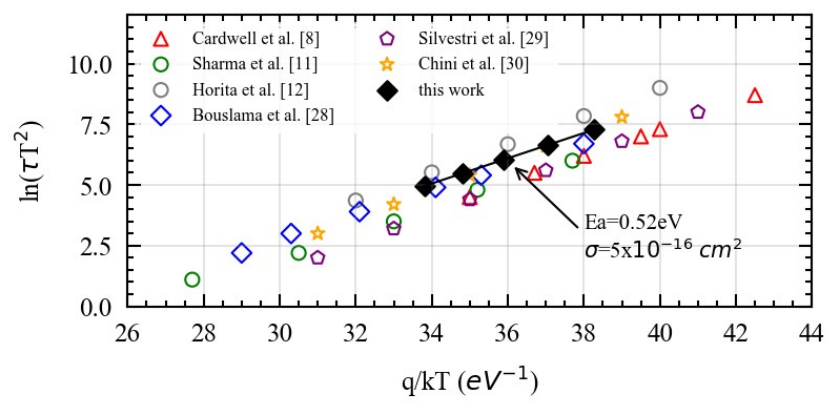

Fig. 7. Comparison between the Arrhenius plot measured in this work and those associated to Fe-related traps by other authors. Points measured in this work well agree to those in the literature, confirming that the observed DCTs are due to electron emission from Fe-traps.

under different measurements conditions, i.e., evaluate the effect of device self-heating and electric field on the emission time constant of said trap.

\section{FE-TRAP EMISSION TIME DEPENDENCE FROM DEVICE BIAS CONDITIONS}

In order to investigate the role of both SHE and electric field on the Fe-trap emission process, the measurement method described in Section II was adopted. A thermal chuck was used for setting the DUTs base-plate temperature at $30{ }^{\circ} \mathrm{C}$ during the measurement, fixing the boundary conditions for the experiments. The induced DCTs have been measured starting from several steady-state biasing conditions, with the aim to span a sufficiently broad range of power levels and voltages. To a first approximation, the applied $V_{\mathrm{DS} \text {,steady }}$ is directly linked to the electric field intensity, whereas the $I_{\mathrm{D} \text {,steady }} \times V_{\mathrm{DS} \text {,steady }}$ product defines the power dissipation within the device. Steady state bias conditions were thus chosen with $V_{\text {DS,steady }}$ ranging from $15 \mathrm{~V}$ to $40 \mathrm{~V}$ with a $5 \mathrm{~V}$ step and dissipated power $\left(P_{\mathrm{D} \text {,steady }}\right)$ ranging from $0.4 \mathrm{~W} / \mathrm{mm}$ to 2.0 $\mathrm{W} / \mathrm{mm}$ with a $0.4 \mathrm{~W} / \mathrm{mm}$ step for a total of 30 different steadystate bias conditions.

The remainder of this section is organized as follows. First, the choice of the DCT measurement conditions adopted for the analysis is explained in detail. Then, results showing the role 
of SHE and electric field for a typical type A device are discussed. Finally, we present results of a systematic analysis on devices of the three types considered.

\section{a) Choice of the Bias Conditions}

Prior to the actual DCT characterization, each steady-state condition was held for a sufficiently long time (more than 2 minutes) to guarantee thermal equilibrium. Furthermore, the trap-filling pulse parameters were chosen to deliberately induce a relatively small current variation $(\sim 4 \mathrm{~mA} / \mathrm{mm})$ to avoid excessive perturbation of the steady-state operating point. This choice was mainly motivated by the following considerations: i) a large current variation would change significantly the dissipated power during the monitored transient [17], whereas a small perturbation does not affect the power set in steady-state condition, thus yielding negligible temperature variations; ii) a small current variation is representative of a low amount of trapped charge variation [30-32], whose dynamics is expected to not significantly alter the electric field profile during the DCT.

Equivalently to Section II, during the short filling pulse (1 $\mu \mathrm{s})$, the devices are biased at $V_{\mathrm{GS}, \text { pulse }}=-3.8 \mathrm{~V}$, i.e., $1 \mathrm{~V}$ below DUT's $V_{\mathrm{TH}}$, so that the subthreshold current flowing through the channel/buffer provides the electrons to be trapped [30] and just the Fe-traps response is induced. The $V_{\mathrm{DS} \text {,pulse }}$ level is instead adjusted for each DCT in order to induce a constant amplitude in the $\mathrm{d} I_{\mathrm{D}} / \mathrm{dlog}_{10} t$ peak for all the acquired DCTs. This choice was adopted to ensure the same variation of trapped charge into buffer states. In fact, since the amplitude of the peak is related to the amount of current dispersion caused by the trap with the considered time constant [30], i.e., to the amount of trapped electrons [31,32], maintaining a constant peak amplitude ensures a constant variation of trapped charge during the filling pulse, regardless of the steady state bias.

\section{b) Role of SHE on emission time constant}

In the experiments reported in Fig. 8, the self-heating effect is evaluated by fixing $V_{\mathrm{DS} \text {,steady }}$ at $15 \mathrm{~V}$ while varying $P_{\mathrm{D} \text {,steady }}$ between $0.4 \mathrm{~W} / \mathrm{mm}$ and $2.0 \mathrm{~W} / \mathrm{mm}$ by varying $I_{\mathrm{D} \text {,steady. }}$. Measured DCTs are represented in terms of current variation $\left(\Delta I_{\mathrm{D}}\right)$ with respect to the steady state current value at the end of the transient $\left(I_{\mathrm{D} \text {,steady }}\right)$ in order to directly compare results obtained at different $I_{\mathrm{D} \text {,steady values. }}$

As we can see in Fig. 8(b), the peak in the $\mathrm{d} I_{\mathrm{D}} / \mathrm{dlog}_{10} t$ plot moves towards shorter times when increasing $P_{\mathrm{D}, \text { steady, }}$ according to the temperature rise in the device active region. The dependence of the emission rate on the SHE is consistent with expectations; in fact, the $\mathrm{CC}$ related to Fe-related Buffer traps is due to electron capture and the resulting DCT is due to the emission of trapped carriers to the conduction band. Since charge emission is a thermally activated process, the increase in the temperature caused by SHE is responsible for the speedup observed while passing from $0.4 \mathrm{~W} / \mathrm{mm}$ to $2.0 \mathrm{~W} / \mathrm{mm}$.

\section{c) Role of electric field on emission time constant}

The electric field effect is shown in Fig. 9. By varying $V_{\mathrm{DS} \text {,steady }}$ in the device's saturation region between $15 \mathrm{~V}$ and 40 $\mathrm{V}$ while maintaining a constant power dissipation $P_{\mathrm{D}, \text { steady }}=0.4$
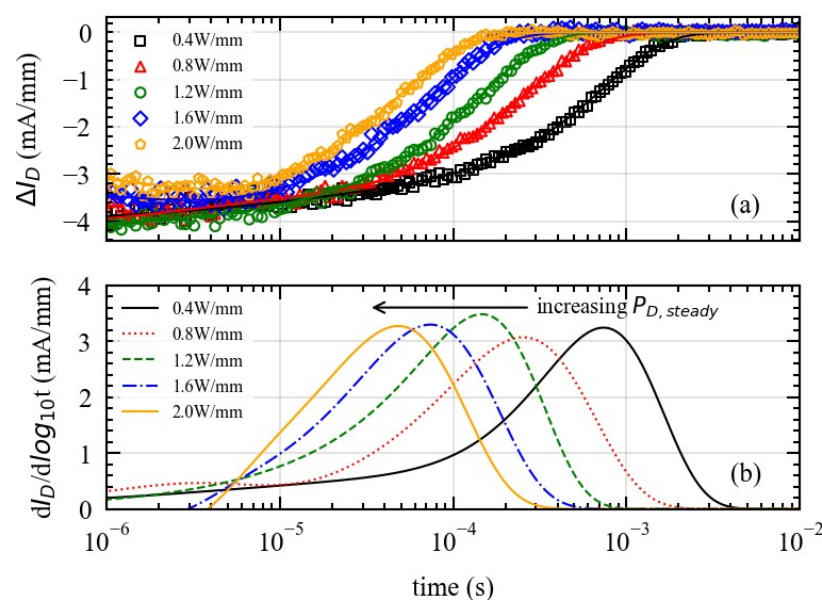

Fig. 8. (a) DCTs measured at constant $V_{D S \text { steady }}=15 V$ (i.e., constant field) at different dissipated power $\left(P_{\mathrm{D}, \text { steady }}\right)$ for device $A$ and (b) corresponding $\mathrm{d} / \mathrm{D} / \mathrm{dlog}_{10}$. The charge emission process speeds-up at high $P_{\mathrm{D} \text {,steady }}$ due to the temperature rise produced by device SHE.
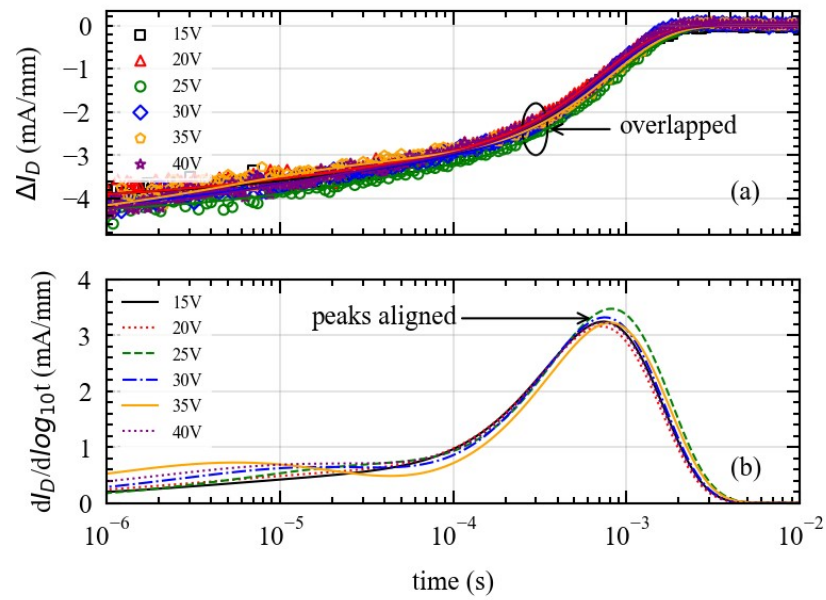

Fig. 9. (a) DCTs measured at constant power $\left(P_{\mathrm{D} \text {,steady }}=0.4 \mathrm{~W} / \mathrm{mm}\right)$ and different $V_{D S \text { steady }}$ for device $A$ and (b) corresponding $\mathrm{d} / \mathrm{D} / \mathrm{dlog}_{10} t$. The field effect is negligible and the derivative peaks are perfectly aligned.

$\mathrm{W} / \mathrm{mm}$ we investigated operating points where the SHE is the same among all the DCTs considered. As a consequence, if the electric field plays any role in the emission time constant, we are expecting some variation at the increase of $V_{\mathrm{DS} \text {,steady. }}$. Fig. 9(a) shows that the measured DCTs at different $V_{\mathrm{DS} \text {,steady }}$ are essentially overlapped and the corresponding peaks in the $\mathrm{d} I_{\mathrm{D}} / \mathrm{dlog}_{10} t$ plot are perfectly aligned (see Fig. 9(b)). Accordingly, we conclude that the emission rate of the $\mathrm{Fe}$ related acceptor is insensitive to the applied $V_{\text {DS }}$ up to $40 \mathrm{~V}$.

\section{d) Time constants from all the steady state conditions}

In order to highlight the generality of the previously discussed SHE and electric field dependencies, the characterization was performed on all the 30 steady-state bias points previously defined. Emission time constant from typical devices of type A, B and C geometries have been extracted, see Fig. 10(a), 10(b) and 10(c), respectively. The emission speed-up produced by SHE is always present, even if this effect is less pronounced for smaller devices, since a reduced periphery yields a lower temperature increase for the same power density. Nevertheless, for all tested devices we can see the same underlying trends. In the explored bias range, the 


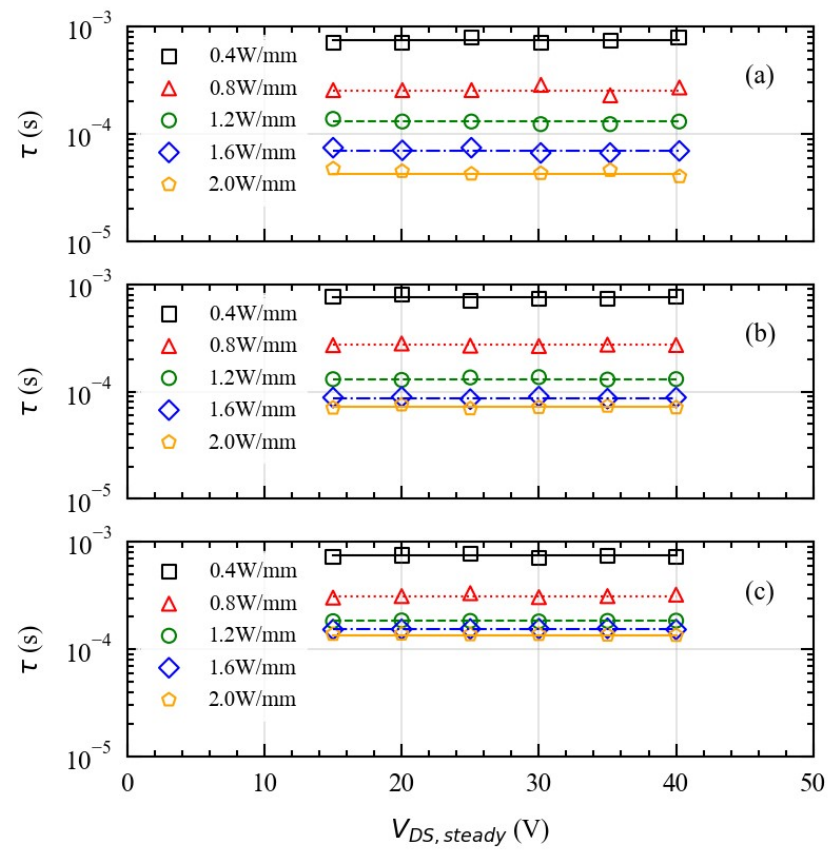

Fig. 10. Emission time constants extracted at several $P_{\mathrm{D} \text {,steady }}$ levels and different $V_{D S, \text { steady }}$ for (a) device A $(10 \times 100 \mu \mathrm{m})$, (b) device B $(8 \times$ $75 \mu \mathrm{m})$ and $(\mathrm{c})$ device $\mathrm{C}(4 \times 75 \mu \mathrm{m})$. A variation on $P_{\mathrm{D} \text {,steady }}$ produces a readable variation on $\tau$, while data points collected at different $V_{D S}$ are horizontally aligned for the same $P_{\mathrm{D} \text {,steady }}$

field effect turned out to be negligible at all dissipated power values, while SHE produces a clear enhancement of the emission process, resulting in parallel lines moving toward shorter times at the increase of dissipated power.

\section{NUMERICAL SimULATIONS}

Experimental data clearly shows that the emission rate is not affected by electric field variations at least within the investigated voltage range. This evidence suggests that electric field plays a minor role in enhancing charge emission from

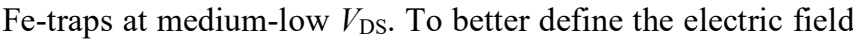
experienced by Fe-traps in our measurements, we carried out 2-D numerical simulations using the commercial Sentaurus (Synopsis Inc.) simulator. Fe-related traps in the GaN buffer were modeled with deep acceptor-like traps at $0.52 \mathrm{eV}$ below the conduction band edge, as experimentally observed by DCTs measurements. The simulation deck was calibrated by comparison with measurements on the devices under examination, see Fig. 11.

Fig. 12 shows the simulated concentration of trapped electrons in the device (e.g., at $V_{\mathrm{DS} \text {,steady }}=40 \mathrm{~V}$ and $P_{\mathrm{D} \text {, steady }}=2.0$ $\mathrm{W} / \mathrm{mm}$ ) from which we observe that the largest concentration of electrons captured by Fe-related traps (i.e., $1 \times 10^{17} \mathrm{~cm}^{-3}$ ) is located under the gate edge towards the drain contact between 150-250 nm from the 2-Dimensional Electron Gas (2-DEG).

Similar profiles were observed for all the bias conditions investigated in this work, indicating that the largest concentration of ionized Fe-traps is typically located in this region, in accordance with previously published results [5]. Particularly, the depth from which the carriers are emitted does not change significantly for the bias conditions explored.

We thus consider the electric field in correspondence to the

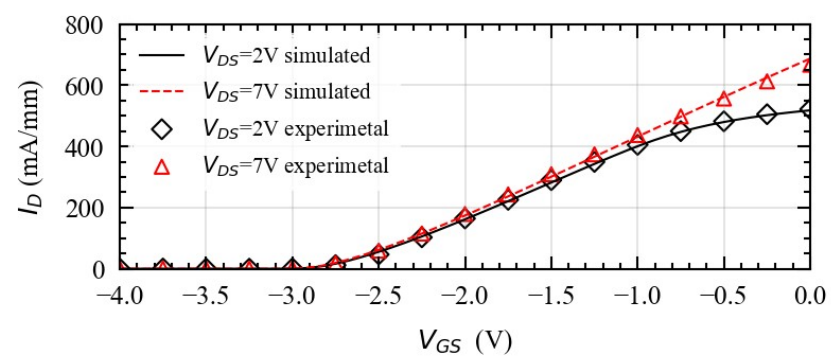

Fig. 11. Comparison between experimental and simulated pulsed ID$V_{G S}$ characteristics obtained at room temperature for tested devices at $V_{D S}=2 \mathrm{~V}$ and $V_{D S}=7 \mathrm{~V}$.

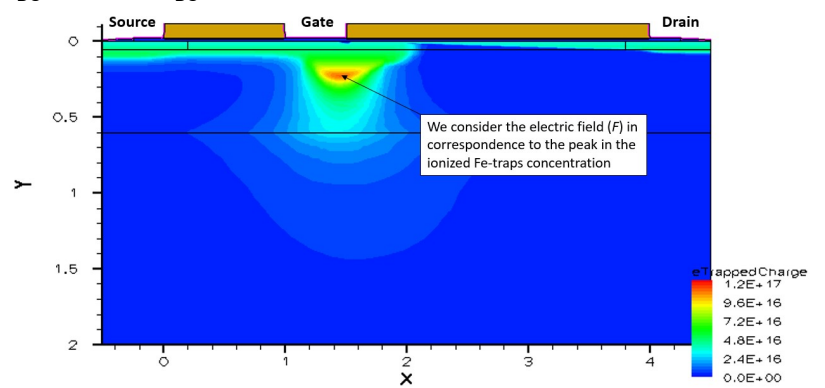

Fig. 12. Concentration of electrons trapped in Fe-traps at $V_{D S}$,steady $=40$ $\mathrm{V}$ and $P_{\mathrm{D}, \text { steady }}=2.0 \mathrm{~W} / \mathrm{mm}$. The largest concentration of trapped carriers is located in the GaN buffer layer under the gate edge towards the drain contact between $150-250 \mathrm{~nm}$ from the 2Dimensional Electron Gas (2-DEG).

peak in the ionized trap concentration as the one experienced by Fe-traps for the analysis related to the expected PFE contribution that will be presented in Section V.

According to the experimental analysis presented in Section III, we extracted the electric field $(F)$ in the region of maximum trapping for $V_{\mathrm{DS} \text {,steady }}$ between $15 \mathrm{~V}$ and $40 \mathrm{~V}$ and $P_{\mathrm{D} \text {,steady }}$ from $0.4 \mathrm{~W} / \mathrm{mm}$ to $2.0 \mathrm{~W} / \mathrm{mm}$. The corresponding values of $F$ are reported in Fig. 13, in which $F$ ranges between $0.226 \mathrm{MV} / \mathrm{cm}$ and $0.408 \mathrm{MV} / \mathrm{cm}$ while passing from $15 \mathrm{~V}$ to $40 \mathrm{~V}$. Particularly, the electric field experienced by Fe-traps increases almost linearly with the applied $V_{\mathrm{DS} \text {,steady, with a }}$ negligible dependence on $P_{\mathrm{D} \text {,steady. }}$

According to this observation, we can more thoroughly interpret the results obtained in Section III as follows. Even though the electric field $(F)$ changes considerably while changing $V_{\mathrm{DS} \text {,steady }}$ between $15 \mathrm{~V}$ and $40 \mathrm{~V}$, this variation does not produce any appreciable effect on the emission time constant of Fe-traps in this range.

\section{Electric FieLd Dependence}

The electric field values obtained with numerical simulations can thus be used to better identify the field-related mechanisms that could affect the traps behavior in the explored bias range. As previously stated, several electric field related effects such as PFE, PAT and/or DT can modify the emission rate. PAT and DT typically occurs at very high electric field and they are unlikely to occur in the investigated bias points. On the other hand, PFE is expected to affect emission rate for low-medium voltage levels [19]. The fact that we do not see any field dependence in our experimental data suggests that the traps involved, i.e., the iron related ones, are not experiencing PFE. In order to gain more insights in this behavior, we perform a simple first-order analysis to 
prove that if PFE would occur, completely different experimental trends would have been observed.

The PFE role in enhancing trap emission rate $(e=1 / \tau)$ is given by [18]:

$$
e=A T^{2} \exp \left(-\frac{E_{a}-\Delta E_{P F}}{k T}\right)
$$

where $k$ is the Boltzmann's constant, $E_{a}$ is the activation energy, $T$ is the absolute temperature while $A$ is a constant referring to the trap emission cross section. The $\Delta E_{P F}$ term in (1) refers to the Poole-Frenkel effect and is defined as [18]:

$$
\Delta E_{P F}=\sqrt{\frac{q^{3} F}{\pi \varepsilon_{0} \varepsilon_{r}}}
$$

where $q$ is the elementary charge, $\varepsilon_{0}$ is the vacuum dielectric constant, $\varepsilon_{\mathrm{r}}$ is the GaN dielectric constant and $F$ is the electric field [22]. Looking at equations (1) and (2) it is clear that at the increase of $F$ the emission rate should increase. The electric field in (2) can be defined according to the values reported in Fig. 13, since we are interested in the electric field experienced by Fe-traps in the explored bias conditions.

By taking the natural logarithm of the ratio between the

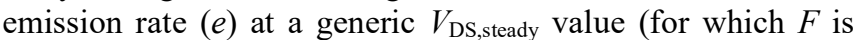
known, according to numerical simulations) and a reference $e_{\text {ref }}$ at $V_{\mathrm{DS}, \text { ref }}=15 \mathrm{~V}$ (i.e., $F_{\text {ref }} \sim 0.226 \mathrm{MV} / \mathrm{cm}$ ), it is now possible to predict the relative $e$ enhancement due to PFE. Furthermore, if we compare data obtained at the same $P_{\mathrm{D}, \text { steady }}$, as it has been done experimentally, the temperature variations are negligible thus allowing to simplifying the $A T^{2} \exp \left(-E_{a} / k T\right)$ term in (1) highlighting in (3) the square root dependence on $F$ :

$$
\ln \left(\frac{e}{e_{r e f}}\right)=\frac{\sqrt{\frac{q^{3}}{\pi \varepsilon_{0} \varepsilon_{r}}}}{k T}\left[\sqrt{F}-\sqrt{F_{r e f}}\right]
$$

Despite this simplification, a realistic value for $T$ (i.e., temperature in the device active region) is still needed to calculate (3). To this end, $T$ was evaluated for device A at several dissipation levels with the McAlister's method [33], yielding a thermal resistance $\left(R_{T H}\right)$ of $22 \mathrm{~K} / \mathrm{W}$ (not shown). The extracted $R_{T H}$ was then used to infer $T$ for the considered bias range (i.e., $P_{\mathrm{D} \text {,steady }}$ between $0.4 \mathrm{~W} / \mathrm{mm}$ and $2.0 \mathrm{~W} / \mathrm{mm}$ ), according to (4):

$$
T=303 K+R_{T H} \times P_{D, \text { stead } y}
$$

Since device A presented a total gate width of $10 \times 100 \mu \mathrm{m}$, we obtained two limit cases for $T: T=311.8 \mathrm{~K}$ for $P_{\mathrm{D} \text {,steady }}=0.4$ $\mathrm{W} / \mathrm{mm}$ and $T=347 \mathrm{~K}$ for $P_{\mathrm{D} \text {,steady }}=2.0 \mathrm{~W} / \mathrm{mm}$. In order to cover the whole temperature range explored in this study, we considered both values of $T$ in our analysis.

A comparison between the emission rate obtained from experimental data in Fig. 10(a) and analytical values computed with (3) is shown in Fig. 14. Interestingly, the comparison shows that the predicted behavior with (3) is completely different from actual data, indicating that PFE is not taking place. Rather than a rigorous comparison, Fig. 14 should be intended as an integrity check for the validity of the speculation that PFE does not take place for iron traps in these devices. In fact, if PFE was actually affecting emission rate, experimental data points would not lie on a horizontal line, but would rather be placed nearby the curves calculated with (3). For this reason, we can conclude that the PFE can be ruled out

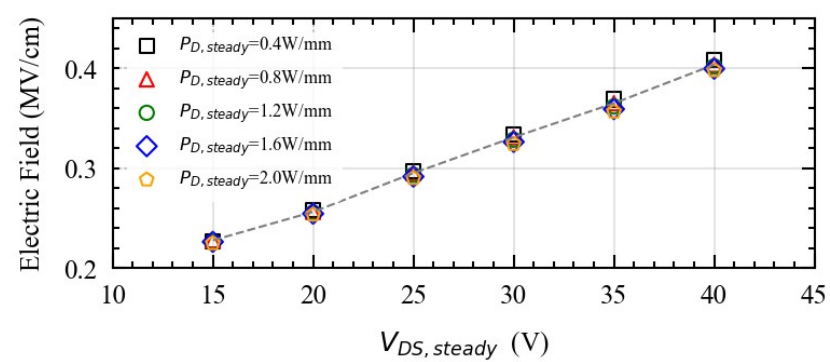

Fig. 13. Electric field $(F)$ values obtained with numerical device simulation for the explored bias conditions. While $F$ increases approximately linearly with the applied voltage $\left(V_{D S \text {,steady }}\right)$, it has negligible dependence on $P_{\mathrm{D} \text {,steady. }}$.

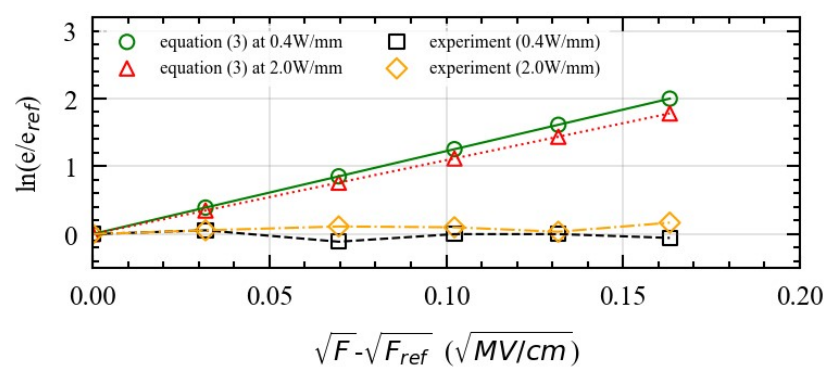

Fig. 14. Relative variation in the emission rate produced by $F$ on device A w.r.t. the field enhancement expected for PFE. The PFE enhancement has been estimated with (4) for $P_{\mathrm{D} \text {,steady }}=0.4 \mathrm{~W} / \mathrm{mm}$ and $P_{\text {D,steady }}=2.0 \mathrm{~W} / \mathrm{mm}$.

as mechanism inducing emission acceleration for Fe-traps, since in the explored voltage range it would have produced a significant $V_{\mathrm{DS}}$ (i.e., field) dependence, which is on the contrary absent. This evidence highlights the nature of iron traps. In fact, for a trap state to experience PFE, it must be charged when empty and neutral when it captures a carrier (i.e., donor-like) [20-22]. Conversely, iron dopants introduce a deep acceptor-like trap which is normally neutral and becomes negatively charged by capturing an electron from the conduction band [34]. The trap potential can be approximated at first-order by a Dirac well [21], for which the barrier lowering responsible for PFE would be negligible in agreement with the presented experimental results.

\section{CONCLUSIONS}

In this paper, we investigated the role of self-heating and electric field on the emission process from iron-related traps in AlGaN/GaN HEMTs for RF applications. The adopted experimental approach, based on the monitoring of drain current transients with either constant dissipated power or constant drain-to-source bias allowed to effectively evaluate separately self-heating and electric field roles. We found that self-heating produces a consistent acceleration in the thermally activated emission process, as a result of the temperature rise in the device active region. On the other hand, the experimental results revealed for the first time no evidence of the Poole-Frenkel effect (PFE), consistently with the nonCoulombic nature of Fe-related buffer traps. Consequently, Poole-Frenkel effect can be excluded for Fe-traps in GaN, corroborating the conclusion that thermal effects dominate the emission dynamics from these traps under conventional operative conditions. This result clarifies the behavior of iron traps in GaN HEMTs for which the absence of PFE can reduce 
the complexity of the simulation models used to interpret the experiments without loss of accuracy.

\section{REFERENCES}

[1] S. S. H. Hsu, C.-W. Tsou, Y.-W. Lian, and Y.-S. Lin, 'GaN-on-silicon devices and technologies for RF and microwave applications', in 2016 IEEE International Symposium on Radio-Frequency Integration Technology (RFIT), Taipei, Taiwan, Aug. 2016, pp. 1-3, DOI: 10.1109/RFIT.2016.7578151.

[2] K. Nakatani, Y. Yamaguchi, Y. Komatsuzaki and S. Shinjo, "MillimeterWave GaN Power Amplifier MMICs for 5G Application," 2019 IEEE International Symposium on Circuits and Systems (ISCAS), Sapporo, Japan, 2019, pp. 1-4, DOI: 10.1109/ISCAS.2019.8702133

[3] S. C. Binari, P. B. Klein, and T. E. Kazior, 'Trapping effects in widebandgap microwave FETs', in 2002 IEEE MTT-S International Microwave Symposium Digest (Cat. No.02CH37278), Seattle, WA, USA, 2002, vol. 3, pp. 1823-1826, DOI: 10.1109/MWSYM.2002.1012217.

[4] Y.-F. Wu, A. Saxler, M. Moore, R. P. Smith, S. Sheppard, P. M. Chavarkar, T. Wisleder, U. K. Mishra, and P. Parikh, '30-W/mm GaN HEMTs by Field Plate Optimization', IEEE Electron Device Lett., vol. 25, no. 3, pp. 117-119, Mar. 2004, DOI: 10.1109/LED.2003.822667.

[5] M. J. Uren, J. Moreke, and M. Kuball, 'Buffer Design to Minimize Current Collapse in GaN/AlGaN HFETs', IEEE Trans. Electron Devices, vol. 59, no. 12, pp. 3327-3333, Dec. 2012, DOI: 10.1109/TED.2012.2216535.

[6] S. Heikman, S. Keller, S. P. DenBaars, and U. K. Mishra, 'Growth of Fe doped semi-insulating $\mathrm{GaN}$ by metalorganic chemical vapor deposition', Appl. Phys. Lett., vol. 81, no. 3, pp. 439-441, Jul. 2002, DOI: 10.1063/1.1490396.

[7] G. A. Umana-Membreno, G. Parish, N. Fichtenbaum, S. Keller, U. K. Mishra, and B. D. Nener, 'Electrically Active Defects in GaN Layers Grown With and Without Fe-doped Buffers by Metal-organic Chemical Vapor Deposition', Journal of Electronic Materials, vol. 37, no. 5, pp. 569-572, May 2008, DOI: 10.1007/s11664-007-0313-3.

[8] D. W. Cardwell, A. Sasikumar, A. R. Arehart, S. W. Kaun, J. Lu, S. Keller, J. S. Speck, U. K. Mishra, S. A. Ringel, and J. P. Pelz, 'Spatiallyresolved spectroscopic measurements of $\mathrm{Ec}-0.57 \mathrm{eV}$ traps in AlGaN/GaN high electron mobility transistors', Appl. Phys. Lett., vol 102, no. 19, p. 193509, May 2013, DOI: 10.1063/1.4806980.

[9] O. Axelsson, S. GustafssonH. Hjelmgren, N. Rorsman, H. Blanck, J. Splettstoesser, J. Thorpe, J. Roedle, and M. Thorsell, "Application Relevant Evaluation of Trapping Effects in AlGaN/GaN HEMTs With Fe-Doped Buffer," in IEEE Transactions on Electron Devices, vol. 63 no. 1, pp. 326-332, Jan. 2016, DOI: 10.1109/TED.2015.2499313.

[10] Y. Jia, Y. Xu, K. Lu, Z. Wen, A.-D. Huang, and Y.-X. Guo, 'Characterization of Buffer-Related Current Collapse by Buffer Potential Simulation in AlGaN/GaN HEMTs', IEEE Trans. Electron Devices, vol. 65 , no. 8, pp. 3169-3175, Aug. 2018, DOI: 10.1109/TED.2018.2849501.

[11] K. Sharma, E. Dupouy, M. Bouslama, R. Sommet, and J.-C. Nallatamby, 'Impact of the Location of Iron Buffer Doping on Trap Signatures in GaN HEMTs', in 2020 International Workshop on Integrated Nonlinear Microwave and Millimetre-Wave Circuits (INMMiC), Cardiff, United Kingdom, Jul. 2020, pp. 1-3, DOI: 10.1109/INMMiC46721.2020.9160114.

[12]Horita, M., T. Narita, T. Kachi, and J. Suda, 'Identification of origin of $\mathrm{EC}-0.6 \mathrm{eV}$ electron trap level by correlation with iron concentration in $\mathrm{n}$ type $\mathrm{GaN}$ grown on $\mathrm{GaN}$ freestanding substrate by metalorganic vapor phase epitaxy', Appl. Phys. Express, p. 5, 2020, DOI:10.35848/18820786/ab9e7c.

[13]M. Meneghini, I. Rossetto, D. Bisi, A. Stocco, A. Chini, A. Pantellini, C. Lanzieri, A. Nanni, G. Meneghesso, and E. Zanoni, 'Buffer Traps in FeDoped AlGaN/GaN HEMTs: Investigation of the Physical Properties Based on Pulsed and Transient Measurements', IEEE Trans. Electron Devices, vol. 61, no. 12, pp. 4070-4077, Dec. 2014, DOI: 10.1109/TED.2014.2364855.

[14]G. Meneghesso et al., 'Reliability of GaN High-Electron-Mobility Transistors: State of the Art and Perspectives', IEEE Trans. Device Mater. Relib., vol. 8, no. 2, pp. 332-343, Jun. 2008, DOI: 10.1109/TDMR.2008.923743.

[15]A. Y. Polyakov and I.-H. Lee, 'Deep traps in GaN-based structures as affecting the performance of GaN devices', Materials Science and Engineering: R: Reports, vol. 94, pp. 1-56, Aug. 2015, DOI: 10.1016/j.mser.2015.05.001.
[16]C. Potier, A. Martin, M. Campovecchio, S. Laurent, R. Quere, J. C. Jacquet, O. Jardel, S. Piotrowicz, and S. Delage, 'Trap characterization of microwave GaN HEMTs based on frequency dispersion of the outputadmittance', in 2014 9th European Microwave Integrated Circuit Conference, Rome, Italy, Oct. 2014, pp. 464-467, DOI: 10.1109/EuMIC.2014.6997893.

[17]A. Chini, F. Soci, M. Meneghini, G. Meneghesso, and E. Zanoni, 'Deep Levels Characterization in GaN HEMTs-Part II: Experimental and Numerical Evaluation of Self-Heating Effects on the Extraction of Traps Activation Energy', IEEE Trans. Electron Devices, vol. 60, no. 10, pp. 3176-3182, Oct. 2013, DOI: 10.1109/TED.2013.2278290.

[18]O. Mitrofanov and M. Manfra, 'Poole-Frenkel electron emission from the traps in AlGaN/GaN transistors', Journal of Applied Physics, vol. 95, no. 11, pp. 6414-6419, Jun. 2004, DOI: 10.1063/1.1719264.

[19] P. A. Martin, B. G. Streetman, and K. Hess, 'Electric field enhanced emission from non-Coulombic traps in semiconductors', Journal of Applied Physics, vol. 52, no. 12, pp. 7409-7415, Dec. 1981, DOI: 10.1063/1.328731

[20]R. B. Hall, 'The Poole-Frenkel effect', Thin Solid Films, vol. 8, no. 4, pp. 263-271, Oct. 1971, DOI: 10.1016/0040-6090(71)90018-6.

[21]P. N. Butcher, N. H. March, and M. P. Tosi, Eds., Crystalline semiconducting materials and devices. New York: Plenum Press, 1986.

[22]H. Morkoç, Handbook of Nitride Semiconductors and Devices: Electronic and Optical Processes in Nitrides, 1st ed. Wiley, 2008.

[23]D. Bisi, M. Meneghini, C. de Santi, A. Chini, M. Damman, P. Brukner, M. Mikulla, G. Meneghesso, and E. Zanoni, 'Deep-Level Characterization in GaN HEMTs-Part I: Advantages and Limitations of Drain Current Transient Measurements', IEEE Trans. Electron Devices, vol. 60, no. 10, pp. 3166-3175, Oct. 2013, DOI: 10.1109/TED.2013.2279021.

[24]J. Joh and J. A. del Alamo, "A Current-Transient Methodology for Trap Analysis for GaN High Electron Mobility Transistors," in IEEE Transactions on Electron Devices, vol. 58, no. 1, pp. 132-140, Jan. 2011, DOI: 10.1109/TED.2010.2087339.

[25]S. L. Zhao, B. Hou, W. W. Chen, M. H. Mi, J. X. Zheng, J. C. Zhang, X. H. Man, Y. Hao, 'Analysis of the Breakdown Characterization Method in GaN-Based HEMTs', IEEE Trans. Power Electron., vol. 31, no. 2, pp. 1517-1527, Feb. 2016, DOI: 10.1109/TPEL.2015.2416773.

[26]D. Bisi, A. Stocco, M. Meneghini, F. Rampazzo, A. Cester, G. Meneghesso, and E. Zanoni, 'High-voltage double-pulsed measurement system for GaN-based power HEMTs', in 2014 IEEE International Reliability Physics Symposium, Waikoloa, HI, USA, Jun. 2014, p. CD.11.1-CD.11.4, DOI: 10.1109/IRPS.2014.6861130.

[27]A. Benvegnù, D. Bisi, S. Laurent, M. Meneghini, G. Meneghesso, D. Barataud, E. Zanoni, and R. Quere, 'Drain current transient and lowfrequency dispersion characterizations in AlGaN/GaN HEMTs', Int. J. Microw. Wireless Technol., vol. 8, no. 4-5, pp. 663-672, Jun. 2016, DOI: $10.1017 / \mathrm{S} 1759078716000398$

[28]M. Bouslama, V. Gillet, C. Chang, J. C. Nallatamby, R. Sommet, M. Prigent, R. Quéré, and B. Lambert, 'Dynamic Performance and Characterization of Traps Using Different Measurements Techniques for the New AlGaN/GaN HEMT of 0.15- $\mu$ m Ultrashort Gate Length', IEEE Trans. Microwave Theory Techn., vol. 67, no. 7, pp. 2475-2482, Jul. 2019, DOI: 10.1109/TMTT.2019.2907540.

[29]M. Silvestri, M. J. Uren, and M. Kuball, 'Iron-induced deep-level acceptor center in GaN/AlGaN high electron mobility transistors: Energy level and cross section', Appl. Phys. Lett., vol. 102, no. 7, p. 073501, Feb. 2013, DOI: $10.1063 / 1.4793196$.

[30]A. Chini, F. Soci, G. Meneghesso, M. Meneghini, and E. Zanoni, 'Traps localization and analysis in GaN HEMTs', Microelectronics Reliability, vol. 54, no. 9-10, pp. 2222-2226, Sep. 2014, DOI: 10.1016/j.microrel.2014.07.085.

[31]O. Mitrofanov and M. Manfra, 'Mechanisms of gate lag in GaN/AlGaN/GaN high electron mobility transistors', Superlattices and Microstructures, vol. 34, no. 1-2, pp. 33-53, Jul. 2003, DOI: 10.1016/j.spmi.2003.12.002.

[32]J. Liu and Z. Huang, 'Mechanism of Buffer-Related Current Collapse in $\mathrm{AlGaN} / \mathrm{GaN}$ HEMT', in 2019 IEEE International Conference on Electron Devices and Solid-State Circuits (EDSSC), Xi'an, China, Jun. 2019, pp. 1-3, DOI: 10.1109/EDSSC.2019.8754456.

[33]S. P. McAlister, J. A. Bardwell, S. Haffouz, and H. Tang, 'Self-heating and the temperature dependence of the dc characteristics of GaN heterostructure field effect transistors', Journal of Vacuum Science \& Technology A: Vacuum, Surfaces, and Films, vol. 24, no. 3, pp. 624-628, May 2006, DOI: 10.1116/1.2172921. 
[34]H. Schroeder, 'Poole-Frenkel-effect as dominating current mechanism in thin oxide films-An illusion?!', Journal of Applied Physics, vol. 117, no. 21 , p. 215103 , Jun. 2015 , DOI: $10.1063 / 1.4921949$. 